\title{
Author Index to Abstracts
}

\author{
Numbers cited refer to abstract numbers)
}

\author{
Abildgaard U. 58 \\ Ábrahám C. 16, 22 \\ Adcock CJ. 27 \\ Adu S, 52 \\ Akino T. 121 \\ Albers MJIJ. 5 \\ Allemand F, 102 \\ Almaas R. 51,110 \\ Anderson CL. 33, 147 \\ Anderson CT. 70 \\ Andersson S. 126, 129 \\ Antillon F. 57 \\ Arachi S, 102 \\ Arrigo T. 29 \\ Artlich A. 9 \\ Ashton MR, 153 \\ Attie K. 28 \\ Aynsley-Green A, 99 \\ Azcona C. 7
}

Baan J. 5

Baarama R. 73

Bagnoli F. 76

Bairam A. 152

Bakici MZ 83

Bakoula C. 35

Bakr A. 74

Balducci R, 30

Bancalari E. 62

Baptista J. 28

Barbazza CM. 50

Barker DJP. 23

Barko E. 139

Barona P. 7

Barrett CT. 70

Barrett D. 119

Bartmann P. 108

Battajon N. 133

Baudin J. 90. 140

Bauer L. 106

Beattie WS. 79

Bechensteen AG, 72

Becker K, 48

Becmeur F. 40

Beratis NG. 75

Berdau W. 86

Berger HM, 125

Bernard O. 47

Betcher JG, 79

Bettendorf M, 45

Bielharz E, 25

Bientz J, 40

Blanco D. 64

Blennow G. 88

Bock I, 149

Bohles H-J, 49

Bordignon S. 133

Bordugo A, 145

Bossano D. 19

Boudjema K. 40

Bracci R. 76

Braillon P. 138

Brämswig JH, 26

Brand $R, 17,113$
Bratlid D. 103

Brendstrup L. 92

Brenton D. 140

Brugman T. 55

Brune T, 56

Brunot P. 40

Brus F. 69.122

Bucci G. 77

Bucher HU. 78, 131

Buckberg GD. 6

Buckley DN, 79

Buckner A. 39

Bühlmeyer K. 3

Buonocore G. 76

Burlina AB. 145

Butturini F. 77

Cabañas F. 64. 136

Cady E, 94

Calaciura F, 29

Carlsson B. 48

Casara G. 133

Cavallo L. 29

Chalon S. 134

Chambon M. 138

Chenard M-P. 40

Chiarelli F. 63

Cinqualbre J, 40

Claris O. 21. 74

Clayton KL. 27

Cooke RWI. 109

Corrigan N, 38

Cotes PM. 72

Cottet-Emard J-M. 152

Crofton PM, 67

Cunningham K. 80

Daac L. 72

DaRiol R. 145

Daval J-L. 15

Davidson F, 101

Davies EG. 7 !

Davis P. 80

Dean HG, 105

De Beuin NC. 55

Decsi T. 148

Degenhart HJ, 55

Delfinno AM, 77

DeLuca F. 29

den Ouden AL. 106

De Potter WP. 12

Devlieger H. 121

Distefano M. 29

Dodge JA, 142

Donzelli F. 50

Dousset B. 53

Dubowitz L. 100

Dubowitz V. 100

Duc G. 78, 127.131

Dunger DB. 27

Eaton DGM, 100

Ebbecke H. 56

Edwards AD. 4

Edwards D. 94
Eggermont E, 121

Elbourne D. 11.89

Elian J-C. 15

Elliott MJ, 4. 65

Elzenga NJ, 122

Ens-Dokkum M. 17

Ens-Dokkum MH, 113

Eronen M. 129

Ewald U. 114

Ewert P. 86

Fallon P. 4

Farnetani MA, 76

Fee F, 38

Feeny DH. 34

Fehlings D. 37

Feillet F. 53

Finn A. 65

Fiore A. 133

Fischbach M. 40

Fischer G, 86

Fischer T. 86

Fisher D. 93

Fisher DJ. 70

Forster H, 49

Frane J. 28

Freeman V. 139

Furlong WJ. 34

Gagnon R. 132

Gallenga PE. 63

Garcia-Alix A. 64, 136

Garcia-Delgado M. 57

Garcia-Foncillas J. 7

Garcia-Velloso MJ, 7

Gauntlett I, 93

Geisert J. 40

Genzel-Boroviczeny O, 43

Ghersi-Egea J.F. 15

Giannini L, 77

Gibson AT, 81, 82

Gibson FM. 71

Gill B, 84, 85

Gill BA, 109

Glover R, 116

Gluckman PD. 18, 19. 25

Gobber D, 50

Gökalp AS. 83

Golden BE. 137

Golden MHN, 137

Golser A, 139

Gonzalez ES, 139

Gordon-Smith EC. 71

Gortner L. 9. 86

Goto M. 33, 147

Gottschalk ME, 33, 147

Grant A. 89

Grauel EL. 127

Gray SA, 79

Green AA. 24

Greisen G, 92. 95

Guan J. 25

Guerra A. 139

Gustafsson J. 114
Haaland K. 124

Haberland H, 49

Hadchouel M. 47

Hågå $P .72$

Hales CN. 23

Hall C. 103

Halliday HL, 142

Hall MA. 153

Halvorsen S, 72

Hammarlund K, 115

Hampsheir R, 52

Hartikainen-Sorri A-L. 36

Hartley R. 105

Harvey D, 2, 52

Haschke F, 139, 143, 146

Hascoët J-M. 152

Hauck G. 8

Haupt R. 96

Heikamp A. 68

Hellström-Westas L. 88

Henriksen O. 104

Hesse V. 49

Hewitt I. 135

Hey EN, 117

Heywood A. 31. 32

Hieronimi G, 127

Hill A, 132

Hille EM. 106

Hintz RL, 28

Hoffmann GF, 46. 48

Hofmann C, 33, 147

Holland BM, 127

Houdkamp E. 125

Howard CV. 59

Huemer C. 139, 143

Hunter S, 117

Ibiwoye MO, 14

Ignarro LJ, 6, 60

Ihnken K, 6

Ikonen RS, 111

Ipsiroglu OS, 87

Isolauri E, I

Jaeck D, 40

Jäger G, 43

Jakobs C, 46

Janas M. 42

Janisch W, 49

Järvelin M-R, 36

Järvenpää A-L, 130

Jobsis-Vanderviliet FF, 123

Johanson A, 28

Johnson A, 11

Johnson Jr RB, 61

Johnsson H, 128

Jones RA. 89

Jonzon A, 128

Jorch G, 56, 98, 127

Kaila M. 1

Káposzta R, 61. 150

Karg V. 146

Kaya R, 83

Keel M. 78 
Kelnar CJH, 67

Kempson C, 39

Khine MM, 59

Khoschosorur G, 144

Kirkbride V, 90

Kirkham FJ, 4

Kirpalani H, 37, 79

Kirubakaran C, 97

Klautz RJM. 5

Klein P, 3

Koh JL, 70

Koletzko B, 148

Kopcsanyi G, 149

Korkman M, 20

Kovács I, 150

Kovács J, 16, 22

Kruse K, 9

Kuhlefelt S, 130

Kuhls E, 93

Kuivalainen E, 126

Kunze D, 96

Kuusela A-LT, 42

Labrune P, 47

Lachmann B, 107

Lago $P, 133$

Lahlou A, 40

Laing I, 116

Lapillonne A, 21, 74, 138

Larizza D, 30

Laroche SM, 12

Larsen PB, 92

Larsson A, 48

Laugier J, 134

Lau M, 93

Laurent T, 128

Law CM, 23

Leth $\mathrm{H}, 104$

Levene MI, 105, 112

Lewin J, 27

Liikanen A, 20

Lilburn M, 140

Linderkamp O, 127

Lindner S, 56

Lindner W, 91

Lindroth M, 88

Liuzzi S, 29

Livolsi A, 40

Lloyd-Thomas A, 4

Lobefalo L, 63

Loesch S, 3

Lokar V, 50

Lorek A, 94, 140

Lou $\mathrm{H}, 104$

Lucchini R, 102

Lundstrøm KE, 92, 95

Luukkainen P, 111

MacKenzie CA, 10

Macnab AJ, 132

Maier RF. 127

Mäkelä-Bengs P, 130

Mäki MJ, 42, 44

Male C, 139, 143

Mallard CE, 18

Mallard EC, 19

Marchal F, 152

Marlow N 99

Maródi L, 61, 150

Martin-Ancel A, 136

Marttinen A, 44

Masszi G, 149

Mastropasqua L, 63

Matsaniotis N, 35

Matthews DR, 27

Mayatepek E, 48

Maynard R, 4

McClure $G, 127,142$

McCormick D, 90
McGill F, 59

McIntosh N, 31, 32, 101

McLoughlin B, 101

Meienreis-Sudau V, 118

Meinck H-M, 46

Melegh B, 149

Menon G, 101

Messina E, 77

Metsä-Ketelä T, 111

Michel E, 26, 56, 98

Michie CA, 2, 52

Middleton D, 49

Miguet D, 21

Milgrom E, 47

Minutillo C. 135

Misrahi M, 47

Moen A. 110

Moison RMW, 125

Möller J, 9

Molnár D, 16, 22, 149

Monin P. 53

Moodley M, 132

Moore J. 39

Morales CG, 131

Morbin G, 50

Moretti C, 77

Morgese G, 63

Moriette G, 127

Morita K, 6

Moser T. 78

Müller AM, 131

Münch G, 91

Municchi G, 30

Mutch L. 11

Myhrman A. 13

Nelle M, 45

Newman AE, 70

Newton S, 39

Nielsen HC, 151

Nocolai T, 91

Obladen M, 107, 127

Odden J-P. 103

Odièvre M, 47

Oetomo SB, 69

Oillet J, 15

Okken A, 69, 73, 122

Olsén P, 13,36

Oozeer R, 100

Orzalesi M. 102

Ottenkamp J, 5

Øyasæter S, 110

Pacioni C, 102

Panagoulias D, 75

Pap M. 149

Paradiso E, 29

Parkin P, 37

Park WS, 123

Parry G, 39

Pasquino AM, 29, 30

Passeri F, 30

Pearse RG, 81, 82

Peebles D. 94

Pellicer A, 64, 136

Péquignot J-M, 152

Pesonen E, 129

Pessenhofer H, 87

Pietschnig B, 143, 146

Pinxteren JA, 12

Piva E. 145

Plötz FB, 68

Pohjavuori M, 129

Pohland F, 66

Polin RA. 61

Polojaz V, 50

Primhak RA, 10

Pryds O, 95, 104
Pucarelli I, 30

Puntis J, 105

Quaba A, 31, 32

Queißer-Luft A, 8

Quero J, 62, 64, 136

Quinn MW, 105

Rabe H. 56

Raivio KO, 129

Rantakallio P, 13, 36

Rating D, 46

Rebuck N, 65

Reichelt KL. 54

Reid M. 142

Reynolds O, 90

Richter J, 7

Ritchie M, 31, 32

Roberts I, 4

Roberts R, 80

Robinson IC, 27

Roche A. 28

Rognum TO, 54

Roland E, 132

Rootwelt T, 103, 110

Rosegger H, 87

Rosenbaum P, 37

Rosenbaum PL. 34

Rot A, 2

Roth S, 90, 94

Rudolph C, 93

Rupprath G, 45

Rushforth JA, 112

Russel ARB, 71

Rustow B, 96, 118

Ruth V, 20

Rutter N, 119

Ruuska TH, 42

Ruys JH. 17

Saeed M, 109

Saia OS, 133

Saigal S, 34

Saliba E, 134

Salle BL, 21, 74, 138

Sallustio GL, 102

Salo MK, 111

Salvadori S 133

Sandbach E, 143

Santos NT, 139

Saritas S, 91

Sarpola A, 36

Sauer PJJ, 141

Saugstad OD, 51, 54, 110

Sbaraglia T, 77

Schall T, 2

Schieffer F, 40

Schmerling DH, 144

Schmidt B, 80

Schreuder AM, 17, 113

Schultz K, 16, 22

Schuster E, 146

Schwarze J, 108

Schwinger E, 9

Scigalla P, 127

Scott M. 38

Sedin G, 115, 128

Segerer H, 107

Semmekrot BA, 127

Serre MA, 53

Sewell AC, 49

Shaffer T, 120

Shahak H, 150

Shaw B, 99

Shaw BNJ, 109

Shaw NJ, 109

Sherman MP, 6, 60

Shiell AW, 23

Sibbons PD, 14, 59

Sierrasesúmaga L, 7

Sierrasúmaga L, 57

Simeoni U, 40

Simpson J, 119

Sjörs G, 115

Skinner JR, 117

Skov L, 92

Smith A, 31, 32

Sökefeld C, 91

Soo KS, 2

Spranger J, 8

Steendijk P, 5

Stenson B, 116

Stephen R, 31

Stevens H, 68

Stevens PA, 96, 118

Stewart A, 11, 90, 140

Stewart M, 38

Stiris TA, 62, 64, 136

Stirling HF, 67

Stoltenberg L, 51, 54

Storm H, 54

Suguihara C. 62

Sundar T, 51,54

Sunehag A, 114

Svenningsen NW, 88

Takei Y, 94

Tan WKM, 18, 19

Tarnow-Mordi W, 39, 116

Teitel D, 93

Temesvári P, 16, 22

Thiel I, 148

Thomson M, 31, 32

Thoresen M, 124

Thorin E, 152

Toft P, 104

Toksoy HB, 83

Torrance GW, 34

Toscano V, 30

Townsend J, 90, 140

Tozzi C, 102

Tröger J, 45

Tschakaloff A, 9

Türkay S, 83

Turner-Gomes S, 80

Tusini-Cottafavi $P, 102$

Valls-i-Soler A, 120

Van Acker KJ, 12

van Bel F, 5

van Gelder W, 107

Van Goudoever JB, 141

Van Lierde S, 121

VanNie A, 37

van Oeveren W, 69

Van Overmeire B, 122

Van Reempts PJ, 12

van't Veen A, 107

Vanura H, 146

van Velzen D, 14, 59

Van Volthoven CAM, 55

van Zoeren-Grobben D, 125 
Volpe MV, 151

von Siebenthal K, 78

Waldhör T, 143

Wales JHK, 81,82

Ward-Platt M, 99

Wardrop CAJ, 127

Waterston T, 24

Watson A. 31, 32

Wattimena JLD, 141
Watts AP, 27

Way C, 80

Weindling M, 84, 85, 99

Wertheim D, 100

Whitelaw A, 11, 58

Wild J, 105

Wild K, 52

Wilkie R, 116

Wilkinson AR, 27

Willan A, 37
Williams CE, 18, 19, 25

Williams HJ, 65

Williams JR, 101

Willis FR, 135

Wilson DC, 142

Wincott E, 89

Winklhofer-Roob BM, 144

Wolf M. 78

Wolf P. 40

Wolfson M, 120
Wright CM, 24

Wu PYK, 123

Wyatt J, 94

Wynn-Jones PA. 70

Zacchello F, 145

Zani S, 76

Zecchino C. 29

Zeller WP. 33. 147

Zernikow B, 98

Zuckerman M. 4 